Gynäk. Rdsch. 1970;10:I-VIII

\title{
Contents, Vol. 10, 1970
}

\section{Inhaltsverzeichnis}

Übersichtsreferate

Cura, C. O.: vide Dubrauszky, V.

Dubrauszky, V. und Cura, C. O. (Würzburg): Proliferative Vorgänge in der Brust-

drüse und ihre Bedeutung bei der Geschwulstbildung

241

Durand, J.-C.: vide Pilleron, J. P.

Jalûvka, V. (Berlin): Herzkrankheiten und mütterliche Mortalität 209

Labeta, C.: vide Pilleron, J. P.

Pilleron, J. P.; Durand, J.-C. und Labeta, C. (Paris): Sezernierende Mamma ohne

Tastbefund - Bericht über 500 Fälle der Fondation Curie. Anatomopathologische

Untersuchungen und therapeutische Indikationen 161

Kurzfassungen von Ze $\gamma$ tschriftenartikeln

Aboulafia, Y.: vide Polishuk, W. Z.

Ackland, Th. H.: vide Stoll, B. A.

Adams, F. H.; Fujiwara, T.; Emmanouilides, G. C. und Räihä, N. (Helsinki):

Phospholipide der Lunge menschlicher Feten und Kinder mit und ohne hyalinen

Membranen 151

Anderson, Anne B. M.: vide Turnbull, A. C.

Anderson, G. V.: vide Clark, D. M.

Andreucci, D.: vide Paula Martins, C. de

Andriole, V. T.: vide Thornton, G. F.

Bala-Gupta, B.: vide Dhall, K.

Barker, W. F.; Sperling, L.; Dowdy, A. H.; Zeldis, L. J. und Longmire,

W. P., jr. (Los Angeles, Calif.): Behandlung nicht palpabler, durch Mammo-

graphie entdeckter Mammakarzinome 104

Bauer, P.: vide Pantlitschko, M.

Beaudry, P. H.: vide Stern, L.

Beer, A. E. (Philadelphia, Pa.): Fetale Erythrocyten im mütterlichen Kreislauf bei 155 Rh-negativen Frauen 52

Bender, S. (Chester): Die Probleme der Frühgeburt 28

Bennington, J. L.: vide Figge, D. C.

Benson, R. C. und Fujikura, T. (Portland, Oreg.): Placenta circumvallata und circummarginata 198

Bertrand, P. V.: vide Crooke, A. C

Blankenship, W. J.: vide Stahlman, M. T.

Bowers, D.G., jr. undRADLAUER, C.B.(Lackland Air Force Base, Tex.): Brustkrebs nach prophylaktischer subkutaner Mastektomie und plastischer Rekonstruktion mit Silastic-Prothesen 69

Brooks, G. G.: vide Lang, E. K. 
Brush, M. G.; Maxwell, R.; Scherrer, J.; Taylor, R. W. undTYE, G. (London):

Versagen der Plazenta und Small-for-dates-Syndrom 289

Brush, M. G.: vide Taylor, R. W.

Butt, W. R.: vide Crooke, A. C.

Inhaltsverzeichnis III

Byrd, E. H., jr.: vide Lang, E. K.

Carlton, M. A. und Hegarty, R. (Isleworth): Die Frühmorbidität des therapeutischen Abortus 11

Celebre, J. A.: vide Mikuta, J. J.

Cervenka, J.: vide Kotásek, A.

Chapman, K. E.: vide Earely, Th. K.

Choyce, J.: vide Goodlin, R.

Christian, C. D.: vide Halbert, D. R.

Clark, D. M. und Anderson, G. V. (Los Angeles, Calif.): Perinatale Mortalität und

Amnionitis 298

Clarkson, A. R.; Meadows, R. und Lawrence, J. R. (Woodville): Akutes Nieren-

versagen post partum - eine generalisierte Shwartzman-Reaktion 301

Crooke, A. C.; Butt, W. R.; Bertrand, P. V.; El Nahas, H. Z.; Hansotia, M. D.

und Morris, R. (Birmingham): Hormonausscheidung bei Gonadotropin-in-

duzierten Schwangerschaften 278

Cummings, D. H.: vide Lang, E. K.

Damon, A.; Damon, S. T.; Reed, R. B. und Valadian, I. (Cambridge, Mass.):

Alter bei der Menarche von Müttern und Töchtern mit einer Betrachtung über

die Zuverlässigkeit der Erinnerung 121

Damon, S. T.: vide Damon, A.

Davis, B. A. (Montreal): Vaginal-Soor in der Privatpraxis 131

Dhall, K.; Dhall, G. I. und Bala-Gupta, B. (Chandigarh): Uterusperforation

mit der Lippes-Schleife

312

Dhall, G. I.: vide Dhall, K.

Diddle, A. W.; Gardner, W. H.; Williamson, P. J. und O’Connor, K. A. (Knox-

ville, Tenn.): Orale kontrazeptive Medikation und vulvovaginale Candidiasis. . 125 Diddle, A. W.: vide Powell, J. M. Dilworth, E.E.: vide Lang, E. K. Donoso, E.: vide Spritzer, E. Dougherty, R. E.: vide Powell, J. M. Dowdy, A. H.: vide Barker, W. F. Dubrauszky, V. und Schwab, J. (Würzburg): Das Problem des Carcinoma in situ

der Endozervix, des Endometriums und der Endosalpinx $\quad 98$

Dunn, J. E. jr.: vide Kashgarian, M.

Earely, Th. K.; Gallagher, J. Q. und Chapman, K. E. (Denver, Colo.) :Brustkrebs

bei Frauen unter $30 \quad 68$

El Nahas, H. Z.: vide Crooke, A. C.

Embrey, M. (Oxford): Prostaglandine zur Wehenstimulierung 268

Emmanouilides, G. C.: vide Adams, F. H.

Farquhar, J. B.: vide Jenkins, D. M.

Ferguson, D. J. (Chicago, 111.): Planung entsprechender Operationen fur Mamma-

karzinom 64

Figge, D. C.; Bennington, J. L. und Schweid, A. I. (Seattle, Wash.): Zervixkarzi- 
nom nach anfänglich negativem und atypischem zytologischem Befund .... 91 Fowler, W. C, jr.: vide Shingleton, H. M. Fraser, R.: vide Weir, R. J.

IV

Inhaltsverzeichnis

Friedberg, Ch. K.: vide Spritzer, R.

Fujikura, T.: vide Benson, R. C.

Fujiwara, T.: vide Adams, F. H.

Gadow, E. C.: vide Queenan, J. T.

Gallagher, J. Q.: vide Earely, Th. K.

Garcia-Bunuel, R.: vide Misenhimer, H. R.

Gardner, W. H.: vide Diddle, A. W.

Garrett, J.; Polse, S. L. und Morrow, J. W. (Los Angeles, Calif.): Hypertonie bei

Ureterobstruktionen 120

Given, F.T., jr. (Norfolk, Va.): Rektum-Scheiden-Fistel 306

Goldstein, D. P.; Johnson, J. P. und Reid, D. E. (Boston, Mass.): Geburtsleitung

bei intrauterinem Fruchttod 205

Goodlin, R.; McLennan, Ch.; Choyce, J.; Lee, R. und Strickler, J. (Palo Alto,

Calif.): Therapeutische Aborte mittels intraamnialer Injektion von hyperosmola-

rer Kochsalzlösung 22

Gray, J.: vide Stahlman, M. T.

Gruber, H. S. und Klaus, M. H. (Cleveland, Ohio): Intermittierende Beatmung mit Maske und Atembeutel 42

Gusdon, J. P.; Leake, N. H.; Prichard, R. W. und Rhyne, A. L. (Winston-Salem, N. C): Fruchtwasser-Antikörpertiter und andere prognostische Parameter bei der Erythroblastose-Diagnostik 202

Halbert, D. R. und Christian, C. D. (Durham, N. C): Amenorrhoe nach Ab-

setzen von Ovulationshemmern 124

Hanson, G. E. und Keettel, W. C. (Iowa City, Iowa): Die Neugebauer-Le-Fort-

Operation 109

Hansotia, M. D.: vide Crooke, A. C.

Harrison, V. C.: vide Heese, V. H. de

Hedberg, H. und Holmdahl, K. (Uddevalla): Beziehung zwischen der Gesundheit

der Mutter und dem intrauterinen Wachstum des Feten 136

Heese, V. H. de; Harrison, V. C.; Klein, M. und Malan, A. F. (Cape Town):

Intermittierende Überdruckbeatmung beim Hyaline-Membranen-Syndrom

(IPPV) 36

Hegarty, R.: vide Carlton, M. A.

Helmrath, Th. A.; Hodson, A. W. und Oliver, Th. K., jr. (Seattle, Wash.): Überdruckbeatmung

bei Neugeborenen: Der Gebrauch einer Gesichtsmaske. ... 38

Hill, J.: vide Thin, R. N. T.

HilleMan, M. R. (West Point, Pa.): Prophylaxe intrauteriner Virusinfektion. ... 53

Hodson, W. A.: vide Helmrath, Th. A.

Holmdahl, K.: vide Hedberg, H.

Horger, E. O. und Hutchinson, D. L. (Pittsburgh, Pa.:) Diagnostischer Wert des

Fruchtwassers 45

Houdek, J.: vide Kotásek, A. 
Hutchinson, D. L.: vide Horger, E. O.

Jenkins, D. M.; Farquhar, J. B. und Oakey, R. E. (Leeds): Oestrogenausscheidung im Urin der Mutter bei Übertragungen 280

Johnson, J. P.: vide Goldstein, D. P.

Inhaltsverzeíchnis

$\mathrm{V}$

Jones, F. S.: vide Powell, J. M.

Juhlyn, L. und Lidén, St. (Uppsala): Der Einfluss von oralen Kontrazeptiva auf das sexuelle Verhalten und auf die Ausbreitung der Gonorrhoe 59

Karp, L.: vide Pauerstein, C. J.

Kashgarian, M. und Dunn, J. E., jr. (Berkeley, Calif.): Die Zeitdauer des intraepithelialen und präklinischen Stadiums beim Plattenepithelkarzinom der Cervix uteri 81

Keettel, W. C.: vide Hanson, G. E.

Kenyon, F. E. (Oxford): Schwangerschaftsunterbrechung aus psychiatrischen Gründen 18

Klaus, M. H.: vide Gruber, H. S.

Klein, M.: vide Heese, V. H. de

Koga, K. und Maeda, K. (Kyushu): Prophylaktische Chemotherapie mit Amethopterin nach Entfernung einer Blasenmole 24

Kotásek, A.; Cervenka, J.; Houe > ek, J. und Pelák, Z. (Prag): Über unsere Er-

fahrungen mit dem «Schlingenabstrich» 191

Lang, E. K.; Simon, K. J.; Cummings, D. H.; Byrd, E. H., jr.; Moore, H. E.;

Tannehill, R. H.; West, W. C, jr.; Tate, W. B.; Brooks, G. G. und Dilworth,

E. E. (Shreveport, La.:) Arteriographie, Luftfüllung des kleinen Beckens und

Lymphangiographie als zusätzliche Hilfsmittel bei der Stadieneinteilung des Kollumkarzinoms 102

Lawrence, J. R.: vide Clarkson, A. R.

Leake, N. H.: vide Gusdon, J. P.

Lee, R.: vide Goodlin, R.

Lehfeldt, H. (New York, N. Y.): Ungewöhnliche Uterusperforation mit einem

neuen Intrauterinpessar 311

Lenz, P. und Meridies, R. (Düsseldorf): Erfahrungsbericht über 107 Boariplastiken 111

Linden, G. (Berkeley, Calif.): Der Einfluss der sozialen Klasse auf das Überleben

von Krebspatienten 76

Lidên, St.: vide Juhlin, L.

Longmire, W. P., jr.: vide Barker, W. F.

Maeda, K.: vide Koga, K.

Malan, A. F.: vide Heese, V. H. de

Malan, A. F.: vide Stahlman, M. T.

Marsac, J.: vide Vachon, F.

Mattes, L. M.: vide Spritzer, R.

Maxwell, R.: vide Brush, M. G.

McLennan, Ch.: vide Goodlin, R.

Meadows, R.: vide Clarkson, A. R.

Melcher, D. H.: vide Thin, R. N. T. 
Meridies, R.: vide Lenz, P.

Metters, J.: vide Taylor, R. W.

Mikuta, J. J. und Celebre, J. A. (Philadelphia, Pa.): Adenokarzinom der Zervix. 71

Misenhimer, H. R. und Garcia-Bunuel, R. (Baltimore, Md.): Versagen der

Schwangerschaftsverhütung mittels Intrauterinpessar und Pilzinfektion des

Feten 60

VI

Inhaltsverzeichnis

Molloy, W. B. (Sydney): Geburten bei kleinen Frauen

138

Moore, H. E.: vide Lang, E. K. Morris, R.: vide Crooke, A. C. Morrow, J. W.: vide Garrett, J.

Muher, S.: vide Pauerstein, C. J.

Nicol, C. S. (London): Gonorrhö bei Frauen 182

Nicol, C. S.: vide Thin, R. N. T.

Oakey, R. E.: vide Jenkins, D. M.

O'Connor, K. A.: vide Diddle, A. W.

Ojala, A.: vide Stenbäck, F.

Oliver, Th. K., jr.: vide Helmrath, Th. A.

Outerbridge, E. W.: vide Stern, L.

Paintin, D. B.: vide Weir, R. J.

Palumbo, L.: vide Shingleton, H. M.

Pantlitschko, M.; Roberg, E.; Weippl, G. und Bauer, P. (Wien): Normalwerte

und Verteilung von Hämoglobin beim Neugeborenen 148

Pauerstein, C. J.; Karp, L. und Muher, S. (Baltimore, Md.): Geburtsleitung nach

Sectio isthmica $\quad 57$

Paula Martins, C. de; Silva Marques, A. M. da und Andreucci, D. (São Paulo):

Richtlinien zur Geburtseinleitung bei verlängerter Schwangerschaft

Pelák, Z.: vide Kotásek, A.

Pepper, F. D., jr.: vide Shingleton, H. M.

Polishuk, W. Z. und Aboulafia, Y. (Jerusalem): Dextran zur Verhinderung von

peritonealen Verwachsungen 114

Polse, S. L.: vide Garrett, J.

Post, D. F. (Philadelphia, Pa.): Perforation des Uterus durch einen Lippes-Loop und partielle. Ausstossung desselben 189

Powell, J. M.; Jones, F. S.; Dougherty, R. E. und Diddle, A. W. (Knoxville,

Tenn.): Zervixkarzinom: Korrelation von Gefrierschnellschnittdiagnose mit

zytologischem und histologischem Befund 94

Prichard, R. W.: vide Gusdon, J. P.

Pytkowicz-Streissguth, A.; Vanderveer, B. B. und Shepard, Th. H. (Seattle,

Wash.): Die geistige Entwicklung von Kindern mit kongenitalem Rubellasyn-

drom 149

Queenan, J. T. und Gadow, E. C. (New York, N. Y.): Akutes und chronisches Hy-

dramnion 292

Radlauer, C. B.: vide Bowers, D. G., jr.

Ramos, A. D.: vide Stern, L.

Räihä, N.: vide Adams, F. H.

Reed, R. B.: vide Damon, A. 
Reid, D. E.: vide Goldstein, D. P.

Rhyne, A. L.: vide Gusdon, J. P.

Roberg, E.: vide Pantlitschko, M.

Robertson, J. I. S.: vide Weir, R. J.

Rossdale, P.D. (Newmarket): Anormales perinatales Verhalten bei Zuchtpferden. 153

Rotheram, E. B., jr. und Schick, S. F. (Pittsburgh, Pa.): Anaerobe, bakterielle,

nicht durch Clostridien bedingte Infektionen bei septischen Aborten

129

Inhaltsverzeichnis VII

Scherrer, J.: vide Brush, M. G.

Schick, S. F.: vide Rotheram, E. B. jr.

Schwab, J.: vide Dubrauszky, V.

Schweid, A. I.: vide Figge, D. C.

Scott-Emuakpor, M. B. (Ibadan): Bakteriengehalt der Luft in Operationsräumen

eines. Stadtspitals 196

Seldon, M.: vide Spritzer, R.

Shepard, F. M.: vide Stahlman, M. T.

Shepard, Th. H.: vide Pytkowicz-Streissguth, A.

Shingleton, H. M.; Fowler, W. C, jr.; Pepper, F. D., jr. und Palumbo, L. (Chapel

Hill, N. C.): Ureterstrikturen nach Therapie des Zervixkarzinoms 73

S $\pi$ mes, Anja; Vartiainen, E. und WidholM, O. (Helsinki): Postoperative Wund-

komplikationen 113

Silva Marques, A. M. da: vide Paula Martins, C. de

Simon, K. J.: vide Lang, E. K.

Sperling, L.: vide Barker, W. F.

Spritzer, R.; Seldon, M.; Mattes, L. M.; Donoso, E. und Friedberg, Ch. K.

(New York, N.Y.): Arrhythmien während der Wehentätigkeit und der Ent-

bindung bei Frauen mit Herzerkrankungen 49

Stahlman, M. T.; Malan, A. F.; Shepard, F. M.; Blankenship, W. J.; Young,

W. C. und Gray, J. (Nashville, Tenn.): Assisierte Unterdruckbeatmung bei

Kindern mit der Hyaline-Membranen-Krankheit 39

Stallworthy, J. (Oxford): Therapeutischer Abort 1

Stenbäck, F. und Ojala, A. (Oulu): Histiochemische Eigenschaften von Fruchtwasserzellen 147

Stern, L.; Ramos, A. D.; Outerbridge, E. W. und Beaudry, P. H. (Montreal):

Künstliche Unterdruckbeatmung: Anwendung bei der Behandlung der Atem-

insuffizienz des Neugeborenen 41

Stickler, J.: vide Goodlin, R.

Stoll, B. A. und Ackland, Th. H. (Melbourne): Behandlung des Brustkrebses bei

alten Frauen 106

Tannehill, R. H.: vide Lang, E. K.

Tapp, J. W.: vide Thin, R. N. T.

Tate, W. B.: vide Lang, E. K.

Taylor, R. W.; Metters, J.; Brush, M. G. und Tye, G. (London): Plazentar-

funktion und Präeklampsie 287

Taylor, R. W.: vide Brush, M. G.

Thin, R. N. T.; Melcher, D. H.; Tapp, J. W.; Nicol, C. S. und Hill, J. (London): 
Diagnose von Trichomonas vaginalis bei der Frau 61

Thornton, G. F. und Andriole, V. T. (West Haven, Conn.): Bakteriurie bei liegendem Dauerkatheter 118

Tree, M.: vide Weir, R. J.

Turan, E. S. (New York, N.Y.): Bakteriologie der Scheide während der Geburt. . 143

Turnbull, A. C. und Anderson, Anne B. M. (Cardiff): Endokrine Faktoren und

Wehenbeginn 274

Tye, G.: vide Brush, M. G.

VIII

Inhaltsverzeichnis

Tye, G.: vide Taylor, R. W.

Vachon, F. und Marsac, J. (Paris): Peritonealdialyse bei septischem Schock mit

Peritonealzeichen 116

Valadian, I.: vide Damon, A.

Vanderveer, B. B.: vide Pytkowicz-Streissguth, A.

Vartiainen, E.: vide Siimes, Anja

Weippl, G.: vide Pantlitschko, M.

Weir, R. J.; Paintin, D. B.; Robertson, J. I. S.; Tree, M.; Fraser, R. und Young,

J. (Glasgow/London): Renin, Angiotensin und Aldosteron in der normalen

Schwangerschaft 284

West, W. C, jr.: vide Lang, E. K.

Widholm, O.: vide Siimes, Anja

Williamson, P. J.: vide Diddle, A. W.

Wurtman, R. J.: vide Zacharias, Leona

Young, J.: vide Weir, R. J.

Young, W..C: vide Stahlman, M. T.

Zacharias, Leona und Wurtman, R. J. (Boston, Mass.): Blindheit und Menarche. 63

Zeldis, L. J.: vide Barker, W. F.

Aus der Geschichte der Gynäkologie

Amundsen, D. W. und Diers, Carol Jean (Washington, D. C): Die Menarche im

klassischen Griechenland und in Rom 154

Diers, Carol Jean: vide Amundsen, D. W.

Prüfungsfragen für Fachärzte

Prüfungsfragen $\quad 79,159$

Richtige Antworten der Fragen $\quad 80,160,240$

S. Karger · Basel · München · Paris · London · New York @ Sydney Arnold-Böcklin-Strasse 25, CH-4000 Basel 11 (Schweiz)

Alle Rechte, insbesondere das der Übersetzung in andere Sprachen, vorbehalten.

Ohne ausdrückliche Genehmigung des Verlages ist es auch nicht gestattet,

diesen Band oder Teile da aaus auf photomechanischem Wege (Photokopie, Míkrokopie) zu

vervielfältigen.

(C)

Copyright 1970 by S. Karger AG, Verlag für Medizin und Naturwissenschaften, Basel

Printed in Switzerland by Buchdruckerei National-Zeitung AG, Basel

Klischees: Sterner \& Co. AG, Basel 